\title{
Vasoactive intestinal peptide decreases MYCN expression and synergizes with retinoic acid in a human $M Y C N$-amplified neuroblastoma cell line
}

\author{
LUCIE CHEVRIER, ANNIE-CLAIRE MEUNIER, STÉPHANIE COCHAUD, \\ JEAN-MARC MULLER and CORINNE CHADÉNEAU \\ Institut de Physiologie et Biologie Cellulaires, Université de Poitiers, \\ CNRS, 40 avenue du Recteur Pineau, Poitiers, F-86022, France
}

Received May 27, 2008; Accepted August 8, 2008

DOI: 10.3892/ijo_00000097

\begin{abstract}
Neuroblastoma is a pediatric tumor which can spontaneously regress or differentiate into a benign tumor. MYCN oncogene amplification occurs in $22 \%$ of neuroblastomas and is associated with poor prognosis. Retinoic acid (RA), a molecule able to induce differentiation and to decrease MYCN expression, is used in the therapy of neuroblastomas. The neuropeptide vasoactive intestinal peptide (VIP) is known to control proliferation or differentiation of numerous cancer cells. In vitro, VIP induces differentiation of neuroblastoma cells. To determine whether VIP could modulate MYCN expression, we carried out real-time quantitative RT-PCR and Western immunoblot analyses in human neuroblastoma SH-SY5Y and IMR32 cells. The results indicated that VIP reduced MYCN mRNA and protein expression, especially in the $M Y C N$ amplified IMR-32 cells, with a maximal and transient decrease by $\sim 50 \%$ after few hours of treatment with VIP at $10^{-6} \mathrm{M}$. This effect was compared to that of RA at $10^{-5} \mathrm{M}$, which induced a diminution of MYCN mRNA expression by $\sim 25 \%$ after few days of treatment. This indicated that VIP and RA display complementary kinetics. Cotreatments showed that VIP and RA had synergistic effects on regulation of expression of MYCN proteins. VIP and RA cotreatments regulated also expression of two MYCN target genes, SKP2 and TP53INP1. These results suggest that VIP, in combination with RA may have a potential therapeutic benefit in neuroblastomas with $M Y C N$ amplification, a genetic abnormality associated with poor prognosis.
\end{abstract}

Correspondence to: Dr Corinne Chadéneau, Institut de Physiologie et Biologie Cellulaires, Université de Poitiers, CNRS, 40 avenue du Recteur Pineau, Poitiers, F-86022, France

E-mail: corinne.chadeneau@univ-poitiers.fr

Key words: vasoactive intestinal peptide, MYCN, neuroblastoma, retinoic acid, differentiation

\section{Introduction}

Neuroblastomas are malignant tumors derived from neural crest. They represent the most common extracranial tumor of childhood. A particular feature of neuroblastomas is their clinical heterogeneity: some neuroblastomas can spontaneously regress or differentiate into a benign form, the ganglioneuroma, while others are not responsive to actual therapies. The 3-year survival ranges from $95 \%$, for neuroblastomas with good prognosis, to only $30 \%$ for tumors with poor prognosis (1). These different clinical behaviours can be explained in part by numerous genetic alterations which occur in neuroblastomas. Among these, a frequent abnormality is amplification of MYCN oncogene which is found in $22 \%$ of neuroblastomas (1). This amplification is associated with advanced stage disease, rapid tumoral progression and poor prognosis $(1,2)$. The role of MYCN in development of neuroblastomas is demonstrated in transgenic mice which overexpress MYCN in cells derived from neural crest and develop neuroblastomas (3). In these animals, administration of MYCN antisense oligonucleotides reduces tumor incidence and tumoral mass (4). $M Y C N$ belongs to the myc proto-oncogene family with $M Y C C$ and $M Y C L$. The MYCN gene encodes two nuclear proteins of 65 and $67 \mathrm{kDa}(5)$ which act as transcriptional activators after binding to the MAX protein (6). In mice and chicken, MYCN is first expressed homogeneously in neural crest and next only in neuroblasts. After birth, MYCN expression decreases and is faintly detected in the adult brain (7).

Vasoactive intestinal peptide (VIP) is a neuropeptide involved in numerous functions including control of proliferation and/or differentiation in normal and tumoral cells (8). VIP is often present in neuroblastomas and its rate increases with the degree of differentiation of these tumors. A good prognosis is associated with VIP-secreting neuroblastomas (9). Expression of high-affinity VIP receptors have long been reported in neuroblastoma cells (10). In vitro, VIP-receptor system expression is stimulated during dibutyryl-cAMP (dbcAMP)-, phorbol ester- or retinoic acid (RA)-induced differentiation of neuroblastoma cells (11). Furthermore, VIP is known to promote differentiation in these cells (12-14). We have demonstrated that VIP 
induced neuritogenesis, a typical characteristic of neuronal differentiation, in the human neuroblastoma SH-SY5Y cell line (15). In these cells, tetradecanoylphorbol 13-acetate (TPA)- or RA-induced differentiation is accompanied by a decreased MYCN expression $(16,17)$. In the present study, we asked whether VIP-mediated differentiation is associated with a down-regulation of MYCN expression in SH-SY5Y cells and in another human neuroblastoma cell line, IMR-32. In the latter, VIP induces differentiation and proliferation arrest $(13,18)$, but contrary to SH-SY5Y cells, IMR-32 is a $M Y C N$-amplified cell line (19). We also compared the effect of VIP on MYCN expression to that of RA, a differentiating agent known to reduce MYCN expression and used in the therapy of some neuroblastomas.

\section{Materials and methods}

Cell culture. SH-SY5Y and IMR-32 cells were routinely seeded at a density of $10^{6}$ cells $/ 25 \mathrm{~cm}^{2}$ flask, in high glucose (4,500 mg/liter) Dulbecco's modified Eagle's medium (DMEM) with GlutaMAX ${ }^{\mathrm{TM}}$ I and sodium pyruvate (Invitrogen, Cergy Pontoise, France), supplemented with $10 \%$ fetal calf serum (FCS) and $100 \mathrm{U} / \mathrm{ml}$ penicillin and $100 \mu \mathrm{g} / \mathrm{ml}$ streptomycin (Invitrogen). Cells were incubated in a humidified $95 \%$ air, $5 \% \mathrm{CO}_{2}$ controlled atmosphere at $37^{\circ} \mathrm{C}$. Medium was changed every 3 or 4 days. Passages were performed once per week, using $1 \mathrm{X}$ trypsin/EDTA (Invitrogen).

Treatments. SH-SY5Y cells were seeded at a density of $3 \times 10^{6}$ cells $/ 25 \mathrm{~cm}^{2}$ flask in the medium described above and grown to subconfluence for 6 days with a renewal of medium on the third day. Serum-containing medium was then replaced with serum-free medium and cells were cultured for 7 days, with a renewal of serum-free medium on the fourth day. Before treatment, medium was changed for serum-free medium supplemented with $0.1 \%$ bovine serum albumin (BSA), fraction V (Invitrogen). The neuropeptide VIP (Neosystem, Stasbourg, France) was prediluted to $10^{-6} \mathrm{M}$ in serum-free medium containing $0.1 \%$ BSA. Cells were treated daily with VIP to a final concentration of $10^{-8} \mathrm{M}$. An equal volume of medium with $0.1 \%$ BSA without neuropeptide was added to control cells.

IMR-32 cells were seeded at a density of $3 \times 10^{6}$ cells $/ 25 \mathrm{~cm}^{2}$ flask for 3 days in medium with $5 \%$ FCS, $100 \mathrm{U} / \mathrm{ml}$ penicillin and $100 \mu \mathrm{g} / \mathrm{ml}$ streptomycin. Before treatment, medium was changed and cells were treated daily with VIP (final concentration of $10^{-6} \mathrm{M}$ ) and/or RA (Sigma-Aldrich, St Guentin-Fallavier, France) (final concentration of $10^{-5} \mathrm{M}$ ), or glucagon (Neosystem) (final concentration of $10^{-6} \mathrm{M}$ ). An equal volume of PBS and/or dimethylsulfoxide (DMSO) was added to control cells.

Micrographs of cells. Video images of IMR-32 cells treated as above with VIP and/or RA for $48 \mathrm{~h}$ were captured using a digital camera connected to a phase-contrast Olympus microscope.

cDNA synthesis. Total RNA was isolated by using the GenElute $^{\text {TM }}$ Mammalian Total RNA kit (Sigma-Aldrich) following the manufacturer's instructions. Total RNA was quantified with GeneQuant II spectrophotometer (Pharmacia). Aliquots of total RNA were treated with $1 \mathrm{U} / \mu \mathrm{g}$ RNA of DNase I Amplification Grade (Invitrogen) according to the manufacturer's instructions, and in the presence of $10 \mathrm{U} / \mu \mathrm{g}$ RNA of RNaseOUT (Invitrogen). After DNase inactivation, RNA was reverse transcribed using random nonamers (SigmaAldrich) and M-MLV Reverse Transcriptase H Minus (Promega, Lyon, France) according to the manufacturer's instruction.

Real-time RT-PCR and quantification. Real-time RT-PCR was carried out with the LightCycler System (Roche Diagnostics, Meylan, France) by using the LightCycler-FastStart DNA Master SYBR-Green I kit as previously described (15). GAPDH and B-actin mRNA levels were used to normalize the mRNA levels between samples. Primers were designed with Primer3 software (http://www.genome.wi.mit.edu/cgibin/primer/primer3_www.cgi) except for GAPDH and B-actin primers (20) and were as follows: (5'-3') MYCN forward: CGACCACAAGGCCCTCAGT, MYCN reverse: TGACCA CGTCGATTTCTTCCT, SKP2 forward: CTCCACGGCAT ACTGTCTCA, SKP2 reverse: GGGCAAATTCAGAGAAT CCA, TP53INP1 forward: CTTCCTCCAACCAAGAACCA, TP53INP1 reverse: GATGCCGGTAAACAGGAAAA. The cycle program for all amplified cDNA was: $5 \mathrm{sec}$ at $95^{\circ} \mathrm{C}, 5 \mathrm{sec}$ at $60^{\circ} \mathrm{C}$ for GAPDH and MYCN or at $65^{\circ} \mathrm{C}$ for $\beta$-actin, SKP2 and TP53INP1 and $10 \mathrm{sec}$ at $72^{\circ} \mathrm{C}$.

Western immunoblot analysis. After treatments, cells were harvested with $1 \mathrm{X}$ trypsin/EDTA, washed twice with cold PBS and resuspended in $10 \mu 1$ per $10^{6}$ cells of ice-cold lysis buffer [10 mM Tris, pH 7.5, 0.5 mM EDTA, pH 8.0, 0.5 mM dithiothreitol (DTT), 0.5\% CHAPS, 10\% glycerol] supplemented with $0.1 \mathrm{mM}$ phenylmethylsulfonylfloride (PMSF) for SH-SY5Y cells and for IMR-32 cells with additional protease inhibitors (Sigma-Aldrich) containing $10 \mu \mathrm{g} / \mathrm{ml}$ aprotinin, $10 \mu \mathrm{g} / \mathrm{ml}$ leupeptin, $1 \mathrm{mM}$ AEBSF, $1 \mu \mathrm{g} / \mathrm{ml}$ bestatin, $1 \mu \mathrm{g} / \mathrm{ml}$ antipain, $1 \mu \mathrm{g} / \mathrm{ml}$ pepstatin. After $30 \mathrm{~min}$ on ice, the samples were centrifuged at $4^{\circ} \mathrm{C}$ for $20 \mathrm{~min}$ at $10,000 \mathrm{x} \mathrm{g}$. For extraction of nuclear proteins, cells were resuspended in $1 \mathrm{ml}$ per $5 \times 10^{6}$ cells of lysis buffer $(10 \mathrm{mM}$ Tris, $\mathrm{pH} 7.4,3 \mathrm{mM} \mathrm{MgCl} 2,10 \mathrm{mM} \mathrm{NaCl}, 0.5 \% \mathrm{NP} 40$ ). After $5 \mathrm{~min}$ on ice and centrifugation for $10 \mathrm{~min}$ at $2,000 \mathrm{xg}$ at $4{ }^{\circ} \mathrm{C}$, the pellet containing nuclei was resuspended in $30 \mu 1$ per $5 \times 10^{6}$ cells of lysis buffer (20 mM HEPES, pH 7.9, 0.3 M $\mathrm{NaCl}, 1.5 \mathrm{mM} \mathrm{MgCl}_{2}, 1 \mathrm{mM}$ DTT, $1 \mathrm{mM}$ PMSF and the same additional protease inhibitors mentioned above) and incubated $30 \mathrm{~min}$ on ice under vigorous shaking. Samples were then centrifuged $20 \mathrm{~min}$ at $10,000 \mathrm{x}$ g at $4^{\circ} \mathrm{C}$. The supernatants were stored at $-80^{\circ} \mathrm{C}$. Protein concentration was determined by using the Bio-Rad DC Protein Assay (Bio-Rad, Marnes la Coquette, France). Total and nuclear proteins were resolved in $8 \%$ SDS-PAGE and electroblotted for $1 \mathrm{~h}$ at $200 \mathrm{~mA}$, onto Immobilon-P membranes (Millipore, St Quentin en Yveline, France). Total proteins on the membranes were detected by Coomassie blue staining. Membranes were blocked overnight at $4{ }^{\circ} \mathrm{C}$, by using $5 \%$ nonfat milk in TBS containing $0.1 \%$ Tween-20 (TBST) and incubated for $1 \mathrm{~h}$ at room temperature with anti-MYCN antibodies diluted in blocking solution. The 
membranes were rinsed $3 \times 10 \mathrm{~min}$ at room temperature in TBST, incubated for $1 \mathrm{~h}$ with the secondary antibody in $5 \%$ milk in TBST and again rinsed with TBST. Antibodies used were: anti-MYCN NCM II 100 (Calbiochem VWR, Fontenay sous Bois, France) 1:50 and secondary antibody anti-mouse IgG, H\&L Chain Specific (goat) peroxidase conjugated (Calbiochem) 1:20000; or anti-MYCN C19 (Santa-Cruz, Santa Cruz, CA, USA) 1:200 and secondary antibody antirabbit IgG, H\&L Chain Specific (goat) peroxidase conjugated (Calbiochem) 1:20000. These anti-MYCN antibodies were previously used in other studies $(21,22)$. After a brief wash in TBS, bound antibodies were revealed by chemiluminescence with the ECL + detection system (Amersham Bioscience, GE Healthcare Europe GmbH, Orsay, France). The ECL and Coomassie blue stainings were quantified by densitometry with the VisioLab 2000 ${ }^{\mathrm{TM}}$ image analyser (Biocom), with measures carried out independently by two investigators. The densitometric measurements of ECL staining were normalized to the corresponding Coomassie blue staining as loading control.

Statistical analyses. Statistical analyses were calculated using GraphPad Prism.

\section{Results}

VIP decreased MYCN mRNA expression in human neuroblastoma cell lines. The effect of VIP on regulation of MYCN mRNA expression was studied by real-time quantitative RT-PCR in two human neuroblastoma cell lines, SH-SY5Y and IMR-32, which have 1.5 and 25 copies of $M Y C N$ oncogene per haploid genome, respectively (19). SH-SY5Y and IMR-32 cells were treated with VIP for $0.5,1,6$ and for $48 \mathrm{~h}$ with a daily treatment. We have previously shown that VIP induced neuritogenesis in serum-starved SH-SY5Y cells. The maximal effect was obtained with $10^{-8} \mathrm{M}$ VIP (15). The same culture and treatment conditions were repeated here. Our results showed that MYCN mRNA expression was slightly reduced after 0.5 - to 48 -h treatments with VIP, with a statistically significant effect after $48 \mathrm{~h}$ (Fig. 1a).

In IMR-32 cells, previous studies have indicated that VIP induced differentiation with a maximal effect on proliferation arrest at $10^{-6} \mathrm{M}(13,18)$. The same treatment conditions were used in the present study. The data from our experiments indicated that 1- and 6-h treatments with VIP decreased transiently and significantly MYCN mRNA expression by 53 and $32 \%$, respectively (Fig. 1b). These results were obtained with GAPDH mRNA as an internal reference. Comparable decreases were observed when $\beta$-actin mRNA was used as another internal reference (data not shown).

To determine whether down-regulation of MYCN mRNA expression was specific to VIP, the effect of glucagon, a neuropeptide which belongs to the VIP family but which does not bind to the same receptors, was tested. IMR-32 cells were treated with glucagon at $10^{-6} \mathrm{M}$ and for $1 \mathrm{~h}$ because the maximal effect of VIP was observed after a 1-h treatment. The results showed that glucagon had no significant effect on MYCN mRNA expression in the IMR-32 cell line (Fig. 1c).

Comparison of MYCN mRNA expression in control SHSY5Y and IMR-32 cells indicated that MYCN mRNA was

\section{a: SH-SY5Y cells}

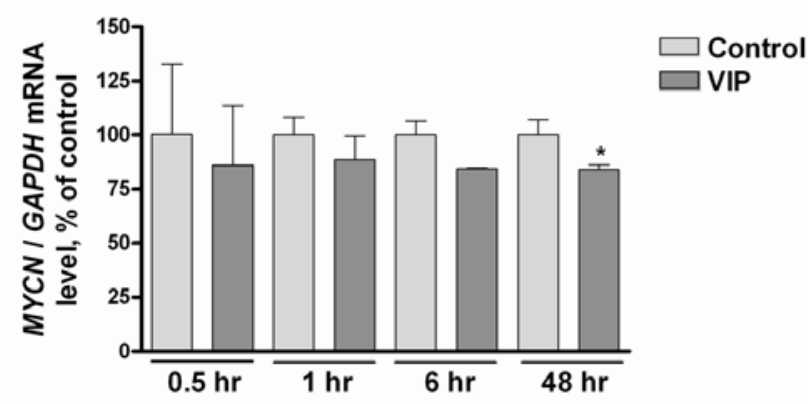

b: IMR-32 cells

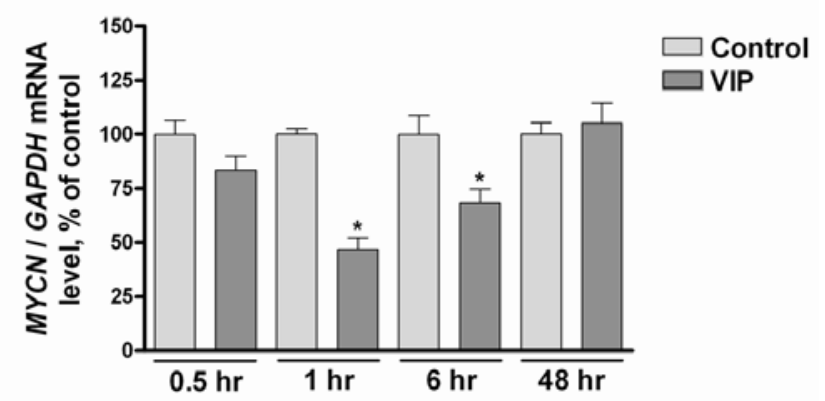

c: IMR-32 cells

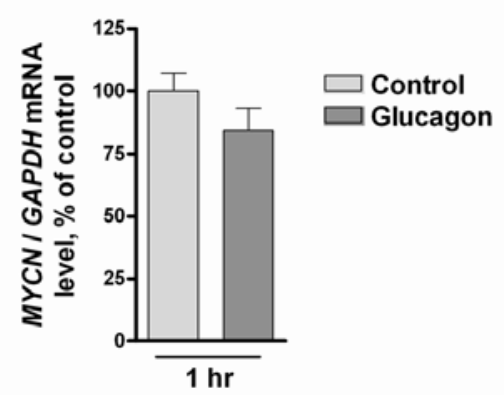

Figure 1. Real-time quantitative RT-PCR analysis of MYCN mRNA in SH-SY5Y cells treated with VIP (a) or in IMR-32 cells treated with VIP (b) or glucagon (c). Total RNA was extracted from SH-SY5Y cells and IMR-32 cells treated or not with VIP at $10^{-8} \mathrm{M}$ and $10^{-6} \mathrm{M}$, respectively, or extracted from IMR-32 cells treated with glucagon at $10^{-6} \mathrm{M}$. VIP-treated cells were cultured for $0.5,1,6$ and $48 \mathrm{~h}$ and glucagon-treated cells were cultured for $1 \mathrm{~h}$. MYCN mRNA expression was quantified by real-time RT-PCR as described in Materials and methods and GAPDH mRNA was used as internal reference. Values are obtained from three independent experiments performed in duplicate and are expressed as percentage of control values. Histograms represent means \pm SEM. The statistical significance was evaluated using a Wilcoxon test $\left({ }^{*} \mathrm{P}<0.05\right)$.

26-fold more expressed in IMR-32 cells than in SH-SY5Y cells (data not shown).

VIP decreased expression of MYCN proteins in human neuroblastoma cell lines. To determine whether VIP-induced down-regulation of MYCN mRNA expression was followed by a decreased expression of MYCN proteins, Western immunoblot analyses were carried out. Two different antiMYCN antibodies were tested. The anti-MYCN NCM II 
a

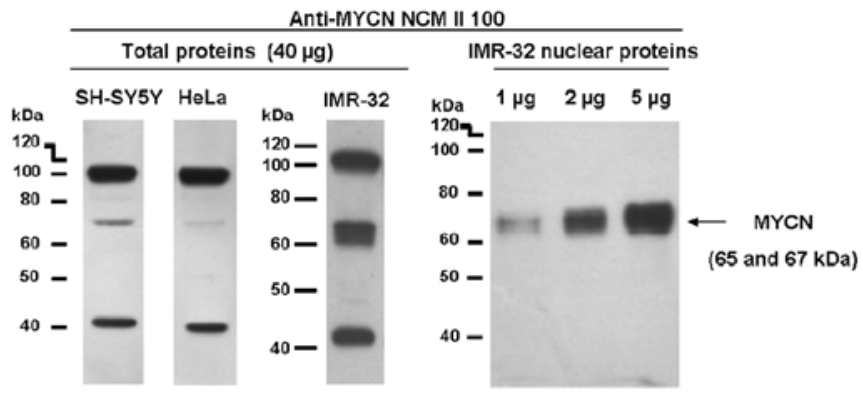

b

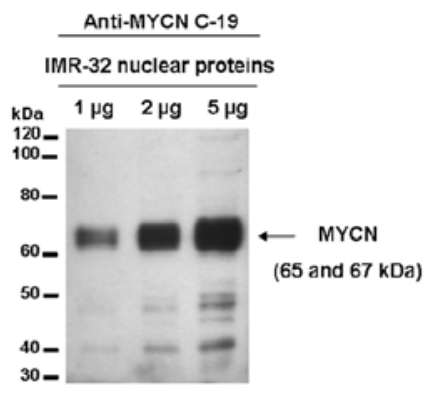

c: SH-SY5Y cells

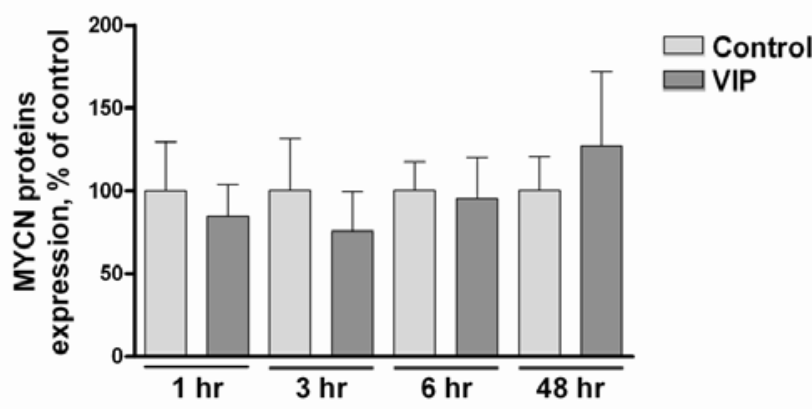

d: IMR-32 cells

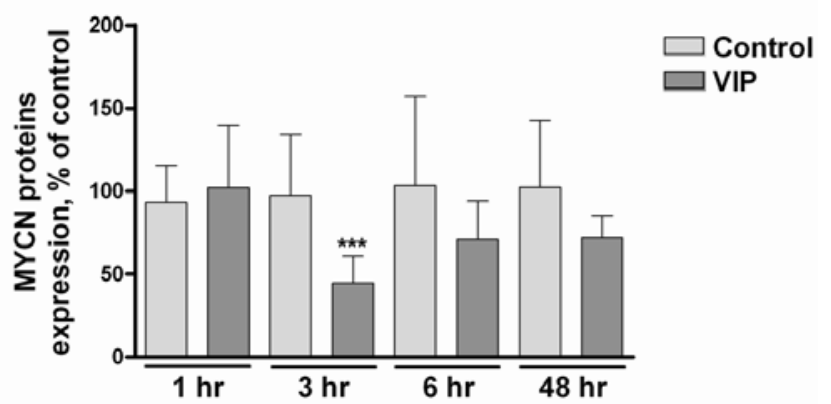

Figure 2. Western immunoblotting of MYCN proteins and quantification analyses of expression of MYCN proteins in SH-SY5Y and IMR-32 cells. Total proteins from SH-SY5Y, IMR-32 and HeLa cells, and nuclear proteins of IMR-32 cells were resolved by SDS-PAGE, transferred to membranes and further probed with the antibody anti-MYCN NCM II 100 (a) or the anti-MYCN C19 (b). Total proteins were extracted from SH-SY5Y (c) and IMR-32 (d) cells treated or not with VIP at $10^{-8} \mathrm{M}$ and $10^{-6} \mathrm{M}$, respectively and cultured for 1, 3, 6 and $48 \mathrm{~h}$. Proteins were analyzed as in (a). The densitometric measurements of $\mathrm{ECl}$ staining were normalized to the corresponding Coomassie blue staining as loading control. Values were obtained from three independent experiments and measures were carried out independently by two investigators. Histograms represent means \pm SEM. Values are expressed as a percentage of corresponding controls. The statistical significance was evaluated using a Wilcoxon test $\left({ }^{* * *} \mathrm{P}<0.001\right)$.

100 antibody (Fig. 2a) detected the expected 65 and $67 \mathrm{kDa}$ proteins but also additional proteins in SH-SY5Y and IMR-32 cells. In the HeLa cell line, known to express trace amount of MYCN proteins (23), only a faint expression of these 65 and $67 \mathrm{kDa}$ proteins was observed and the additional proteins were as well represented as in neuroblastoma cells. In nuclear protein extracts from IMR-32 cells, only the 65 and $67 \mathrm{kDa}$ proteins were present, in agreement with the known localization of this transcription factor. These two proteins were also detected with another anti-MYCN antibody (C-19) in the nuclear extract, together with smaller proteins faintly labelled (Fig. 2b). The anti-MYCN NCM II 100 was used in the next Western immunoblotting experiments in which the 65 and $67 \mathrm{kDa}$ proteins were analyzed by densitometry.

In comparison with control cells, expression of MYCN proteins decreased faintly and transiently in VIP-treated SHSY $5 Y$ cells, with a maximal effect of $24 \%$ after a $3-\mathrm{h}$ treatment which was not statistically significant (Fig. 2c). In the IMR-32 cell line, VIP reduced expression of MYCN proteins from $3 \mathrm{~h}$ of treatment, with a maximal and statistically significant diminution of 54\% after a 3-h treatment (Fig. 2d). Thus, the faint or important down-regulation of MYCN mRNA expression observed in VIP-treated SH-SY5Y or IMR-32 cells, respectively, is followed by a reduction of expression of MYCN proteins, especially in IMR-32 cells which present a higher number of $M Y C N$ copies than SHSY5Y cells. Hence, further experiments were focused on the IMR-32 cell line.

Comparison of effects of VIP and RA on cell morphology and on MYCN mRNA expression in IMR-32 cells. The well known differentiating agent RA has been reported to decrease MYCN expression in neuroblastoma cells after several days of treatment $(17,24)$. However, little is known about the shortterm effects of RA on MYCN expression. To compare the action of VIP to that of RA, we tested two retinoid isomers: 9-cis RA (9-RA) and all-trans RA (at-RA), in the IMR-32 cell line. It was previously shown that these two isomers had different effects on expression of differentiation biomarkers in IMR-32 cells (25). IMR-32 cells were treated daily with 9RA or at-RA at $10^{-5} \mathrm{M}$ for $0.5,1,6,48,72$ and $96 \mathrm{~h}$. These molecules induced formation of cell aggregates and/or neuritic extensions, as previously described in RA-treated IMR-32 cells (13). Compared to control cells (Fig. 3a), a 48-h treatment with at-RA (Fig. 3e) led only to formation of cell aggregates, while 9-RA (Fig. 3c) induced both cell aggregates and neuritogenesis. A 48-h treatment with VIP induced neuritogenesis (Fig. 3b).

The effect of RA isomers on MYCN mRNA expression was then studied by real-time quantitative RT-PCR (Fig. 4a). 


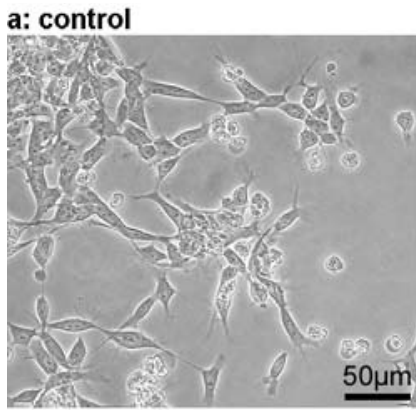

\section{c: 9-RA}

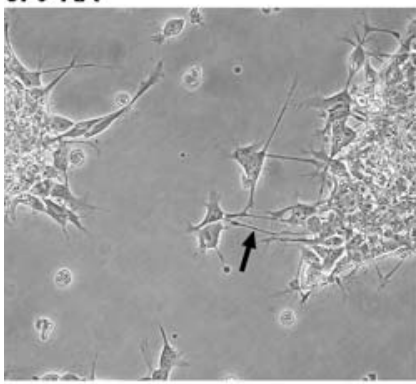

e: at-RA

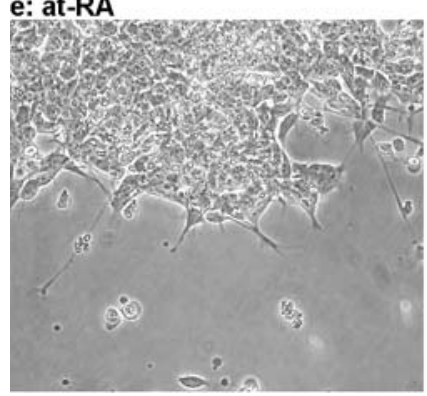

b: VIP

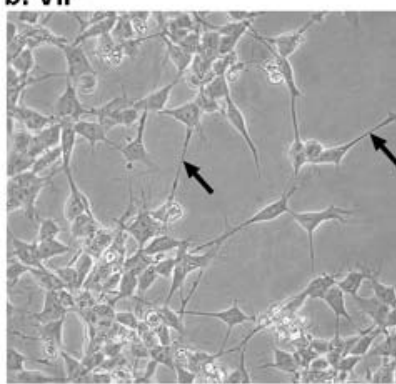

d: VIP + 9-RA

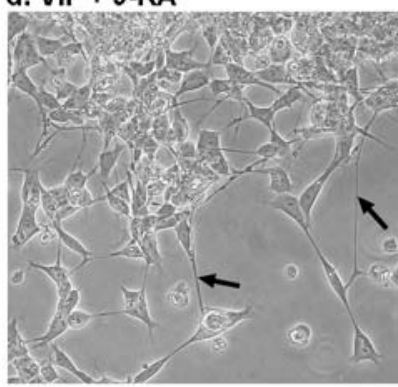

f: VIP + at-RA

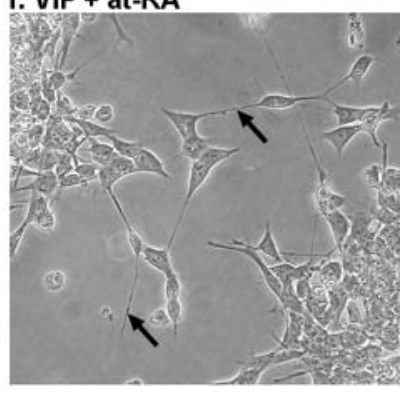

Figure 3. Phase-contrast micrographs of IMR-32 neuroblastoma cells cultured in the presence or absence of VIP and/or RA at $10^{-6} \mathrm{M}$ and $10^{-5} \mathrm{M}$, respectively, for $48 \mathrm{~h}$. Control cells (a) after $48 \mathrm{~h}$ without differentiating agents had few neurites while after $48 \mathrm{~h}$ in the presence of VIP (b) cells extended neurites (arrows). Cells treated with 9-RA (c) exhibit some neurites and form aggregates, while a 48-h treatment with at-RA (e) induces only cell aggregate formation. Cells cotreated with VIP and 9-RA (d) or with VIP and at-RA (f) extended neurites as cells treated with VIP. The scale bar indicated in control cells corresponds to $50 \mu \mathrm{m}$ and applies to all micrographs.

The data indicated that after a 0.5 -h treatment, both RA isomers decreased MYCN mRNA expression by $\sim 23 \%$, an effect which could not be observed after 1 and $6 \mathrm{~h}$ of treatment. A statistically significant increased expression of MYCN mRNA was even induced after a 6-h treatment with 9-RA. After 48- and 72-h treatments, MYCN mRNA expression was reduced by both RA isomers with a maximal and statistically significant lowering of $30 \%$ after a 72-h treatment with 9-RA. After a 96-h treatment, MYCN mRNA expression was decreased only by at-RA. Thus, 9-RA and at-RA did not exactly display the same effects on MYCN mRNA expression. To compare these results with those obtained for VIP treatment, the kinetic of VIP effect in IMR-32 cells shown in Fig. 1b was complemented with longer treatment times. This indicated that 72- and 96-h stimulations by VIP did not modify MYCN mRNA expression (data not shown). It was observed that VIP and RA displayed complementary kinetics of down-regulation of MYCN mRNA expression. Indeed, VIP decreased MYCN mRNA expression only after 1- and 6-h treatments, whereas at least one of the RA isomers reduced

\section{a: RA treatments}

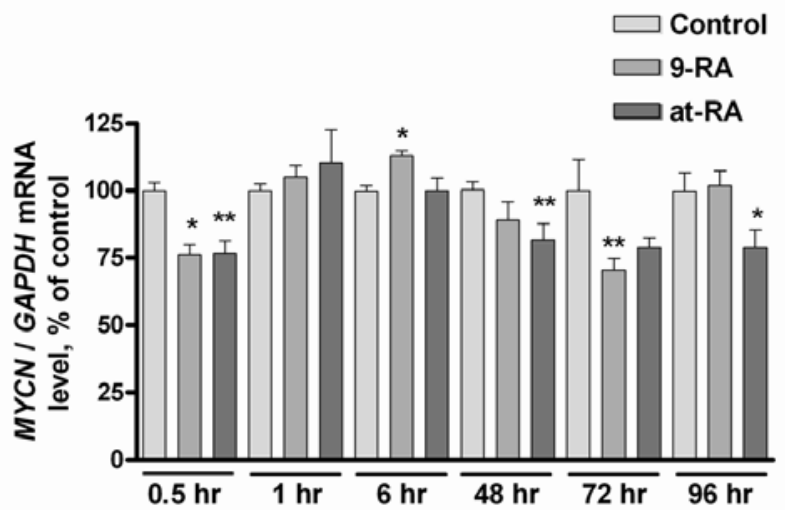

\section{b: cotreatments}
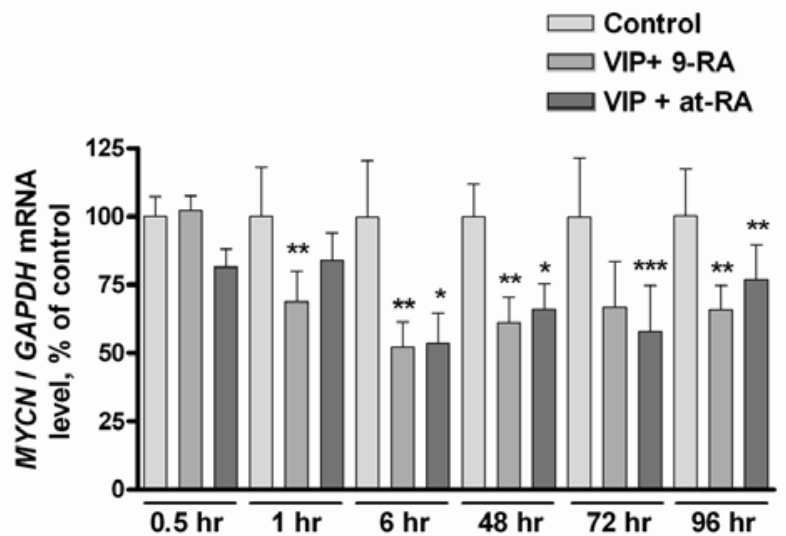

Figure 4. Real-time quantitative RT-PCR analysis of MYCN mRNA in IMR-32 cells treated with RA (a) or cotreated with VIP and RA (b). Total RNA was extracted from IMR-32 cells treated with 9-RA or at-RA at $10^{-5} \mathrm{M}$ and VIP at $10^{-6} \mathrm{M}$ and cultured for $0.5,1,6,48,72$ and $96 \mathrm{~h}$. MYCN mRNA expression was quantified by real-time RT-PCR as described in Materials and methods and GAPDH mRNA was used as internal reference. Values were obtained from two independent experiments performed in quadruplicate. Histograms represent means \pm SEM. Values are expressed as a percentage of corresponding controls. The statistical significance was evaluated using a Friedman test followed by a Dunn's test $\left({ }^{*} \mathrm{P}<0.05,{ }^{* *} \mathrm{P}<0.01\right.$ and $\left.{ }^{* * *} \mathrm{P}<0.001\right)$.

MYCN mRNA level after $0.5,48,72$ and $96 \mathrm{~h}$ but not after 1 and $6 \mathrm{~h}$ of treatments.

Effects of cotreatments with VIP and RA on cell morphology and on MYCN expression in IMR-32 cells. We next studied the combined effects of the differentiating agents VIP and RA. IMR-32 cells were treated daily with VIP at $10^{-6} \mathrm{M}$ together with 9-RA or at-RA at $10^{-5} \mathrm{M}$ for $0.5,1,6,48,72$ and $96 \mathrm{~h}$. After $48 \mathrm{~h}$ of cotreatment, cells exhibited neuritic elongation (Fig. 3d and f) and formed aggregates but these latter were less well-delimited than those induced by RA alone.

The results of real-time quantitative RT-PCR showed that the association of VIP and RA led to a decrease of MYCN mRNA expression from 0.5 to $96 \mathrm{~h}$ (Fig. 4b). Slightly higher effects were often observed with VIP/9-RA cotreatments compared with VIP/at-RA cotreatments, with at least a lowering of $30 \%$ for each time of treatment from 1 to $96 \mathrm{~h}$ and a maximal reduction of $48 \%$ after a 6-h treatment with VIP/9-RA stimulation. The reduction of MYCN/GAPDH 
a

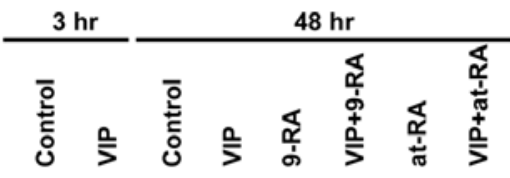

$80 \mathrm{kDa}-$

$60 \mathrm{kDa}-$
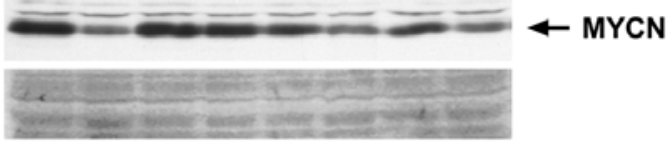

b

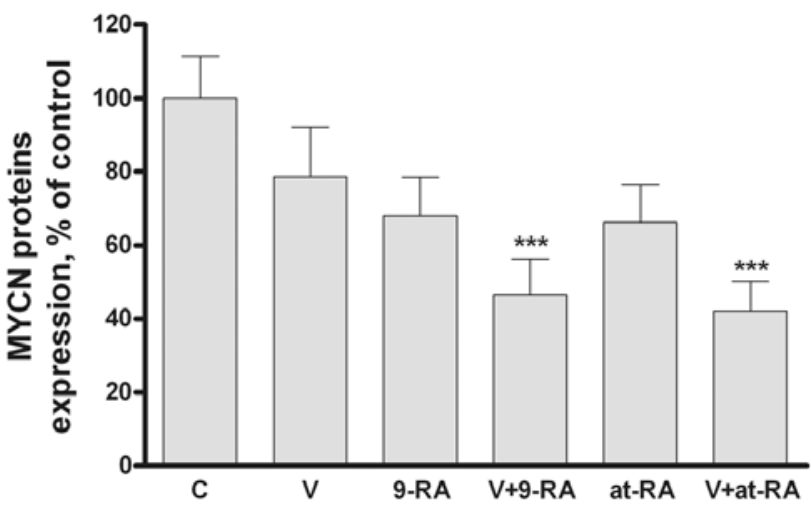

Figure 5. Western immunoblotting of MYCN proteins (a) and quantification analysis (b) of expression of MYCN proteins in IMR-32 cells treated with VIP and/or RA. (a) Total proteins of IMR-32 cells treated as in Fig. 4 and cultured for 3 and $48 \mathrm{~h}$, were resolved by SDS-PAGE, transferred to membranes and probed with the anti-MYCN NCM II 100 antibody. (b) Quantification was performed as in Fig. 2. Values were obtained from two independent experiments. Histograms represent means \pm SEM. Values are expressed as a percentage of corresponding controls. The statistical significance was evaluated using a Friedman test followed by a Dunn's test $\left({ }^{* * *} \mathrm{P}<0.001\right)$.

mRNA level was confirmed by using the B-actin mRNA to normalize MYCN mRNA level (data not shown). The combination of VIP and RA induced a decrease of MYCN mRNA expression which appeared early after the beginning of treatment, and was more lasting and more important than RA- or VIP-induced down-regulation, suggesting a synergy between VIP and RA.

To determine whether VIP and RA could act in synergy to regulate expression of MYCN proteins, Western immunoblot analysis was performed. This analysis was carried out after $48 \mathrm{~h}$, a treatment time after which VIP or RA alone had no or weak effect on MYCN mRNA expression, contrary to VIP/RA cotreatments which decreased MYCN mRNA level by $\sim 40 \%$. The data indicated that VIP or the RA isomers alone reduced expression of MYCN proteins by $30 \%$ (25, 33 and $36 \%$ for VIP, 9-RA and at-RA, respectively), while cotreatments amplified this down-regulation, reaching $\sim 60 \%$ (55 and $59 \%$ for VIP/9-RA and VIP/at-RA, respectively) (Fig. 5). For comparison, the effect of a 3-h treatment with VIP which induced a maximal lowering of expression of MYCN proteins of 54\% (Fig. 1b) is shown (Fig. 5a, lanes 1 and 2). Thus, VIP and RA acted in synergy to decrease expression of MYCN proteins in the IMR-32 cell line after a 48-h treatment.

Effects of VIP and/or RA on expression of MYCN target genes in IMR-32 cells. Finally, we tested whether the decrease in a: SKP2 mRNA after a 6-hr treatment

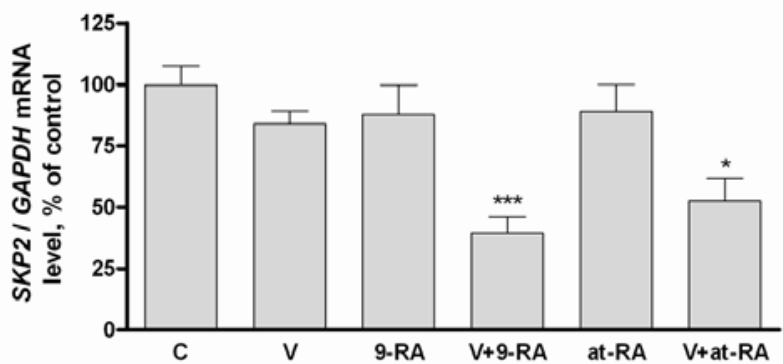

b: SKP2 mRNA after a 48-hr treatment

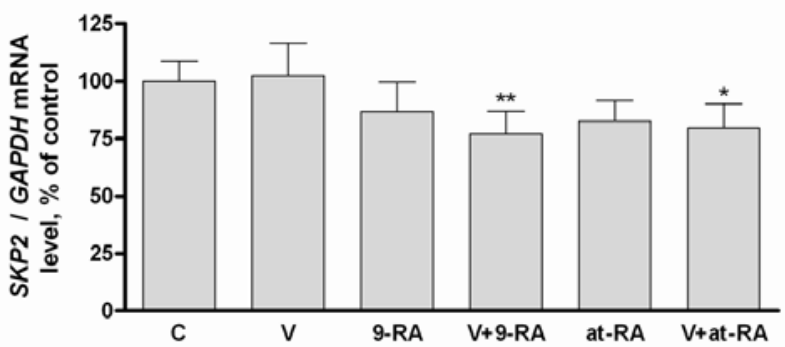

c: TP53INP1 mRNA after a 72-hr treatment

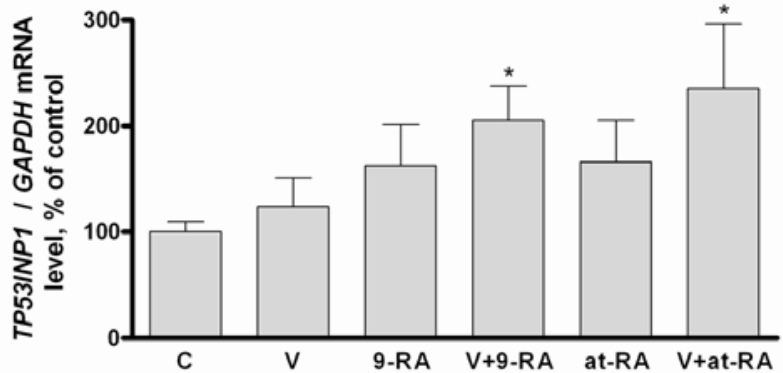

Figure 6. Real-time quantitative RT-PCR analysis of SKP2 mRNA and TP53INP1 mRNA in IMR-32 cells cultured in the presence or absence of VIP and/or RA. Total RNA was extracted from IMR-32 cells treated with VIP and/or RA at $10^{-6}$ and $10^{-5} \mathrm{M}$, respectively. SKP2 mRNA expression after 6 (a) and $48 \mathrm{~h}$ (b) of treatment and TP53INP1 mRNA expression after $72 \mathrm{~h}$ (c) of treatment were quantified by real-time quantitative RT-PCR as described in Materials and methods. Values were obtained from two independent experiments performed in quadruplicate. Histograms represent means \pm SEM. Values are expressed as a percentage of corresponding control. The statistical significance was evaluated using Friedman followed by a Dunn's test $\left({ }^{*} \mathrm{P}<0.05,{ }^{* *} \mathrm{P}<0.01\right.$ and $\left.{ }^{* * *} \mathrm{P}<0.001\right)$.

expression of the transcription factor MYCN induced by VIP/RA cotreatments affected expression of MYCN target genes. In a recent study, several genes involved in cell cycle were identified as being regulated by MYCN. This was shown in IMR-32 cells by microarrays after MYCN RNA interference and confirmed by real-time quantitative RT-PCR (26). Among these genes, SKP2 (S-phase kinase-associated protein 2) was down-regulated early after the decrease in MYCN expression, while expression of TP53INP1 (tumor protein p53 inducible nuclear protein 1) was up-regulated late after MYCN knockdown. Thus we studied mRNA expression of SKP2 and TP53INP1 after 6- and 72-h treatments, respectively, in IMR-32 cells treated with VIP and/or RA. The data from 
real-time quantitative RT-PCR indicated that SKP2 mRNA expression was only slightly reduced after a 6-h treatment with VIP or RA alone (Fig. 6a), whereas cotreatments induced a statistically significant lowering, with a maximal effect of $60 \%$ after VIP/9-RA cotreatment. Although reduced, this effect was still observed after a 48 -h cotreatment at least for VIP/9-RA that decreased more efficiently the SKP2 mRNA level than 9-RA alone (Fig. 6b). For TP53INP1, the data indicated that, after a 72-h treatment, VIP and RA alone or in cotreatment tended to elevate expression of this mRNA (Fig. 6c). This effect was more important and statistically significant with cotreatments. Compared to control cells, cotreatments increased TP53INP1 mRNA expression by $\sim 2$-fold.

\section{Discussion}

The data presented in this study indicate that, in the MYCNamplified IMR-32 cell line, VIP decreases MYCN expression at the mRNA and protein levels. Moreover, we found that VIP and RA had synergistic effects on regulation of expression of MYCN proteins and on two MYCN target genes, SKP2 and TP53INP1.

In the present study, we also studied the effect of VIP in the non-amplified SH-SY5Y cell line, for which the neuropeptide had little effect on MYCN expression. We observed that MYCN proteins are less expressed in SHSY5Y cells than in IMR-32 cells, according to the MYCN mRNA level which is much higher in IMR-32 cells. This difference in MYCN expression level between these two cell lines is in quite a good agreement with the known number of MYCN gene copies: 1.5 and 25 per haploid genome, in SHSY5Y and IMR-32 cells, respectively (19). The limited effect of VIP observed in SH-SY5Y cells could be due to the faint level of MYCN expression in this cell line.

During RA-induced differentiation of neuroblastoma cells, morphological differentiation and cell cycle arrest follow the decrease in MYCN expression (27). In IMR-32 cells, we showed that the effect of VIP on MYCN expression was rapid since a maximal down-regulation of MYCN mRNA occurred after a 1-h treatment, while neuritogenesis was well established after $48 \mathrm{~h}$ of stimulation. Thus, VIP, as well as $\mathrm{RA}$, induced reduction in MYCN expression, which is an early event that precedes morphological differentiation.

In the IMR-32 cell line, we noted that 9-RA induced neuritogenesis and cell aggregates, whereas at-RA led only to formation of cell aggregates. These observations are in agreement with studies showing that 9-RA is more efficient than at-RA in promoting morphological differentiation in neuroblastoma cell lines (28-30). In our experiments, this differential effect may be explained by expression of two classes of RA receptors in IMR-32 cells: RA receptors (RAR) and Retinoid X receptors (RXR) (25). Formation of cell aggregates could be mediated by RAR, which bind both RA isomers tested, while neuritogenesis could involve 9-RA selective RXR.

At the molecular level, we observed that MYCN mRNA expression was first decreased after a 0.5 -h treatment with 9-RA, next raised after a 6-h treatment and then was reduced again after 48 and $72 \mathrm{~h}$ of treatment. Thus, 9-RA has opposite effects on MYCN mRNA expression according to treatment time. Such observations indicated that complex regulation governs MYCN expression over time, suggesting the interest in studying short- and long-term effects of differentiating agents. Similar time-dependent effects of RA have already been shown in SH-SY5Y cells: after a 1-h treatment, invasion capacity is increased, while a 96-h treatment inhibits invasiveness (31). In previous studies, RA-induced decrease of MYCN expression was generally studied after few days of treatment.

MYCN proteins are transcription factors which regulate expression of numerous genes. We tested two of these genes, SKP2 and TP53INP1. SKP2 belongs to the SCF ${ }^{\text {spk2 }}$ complex which is responsible for polyubiquitylation of cell cycle inhibitors such as $\mathrm{p} 21^{\mathrm{WAF} 1}(32)$. TP53INP1 induces cell cycle arrest in G1 phase and enhances p53-mediated apoptosis (33). After MYCN RNA interference in IMR-32 cells, it was demonstrated that SKP2 mRNA expression was lowered by $50 \%$ and that TP53INP1 mRNA level was increased with a maximal effect of 7-fold (26). In our experiments, a comparable reduction of SKP2 mRNA expression was induced by VIP and RA cotreatments. Stimulation of TP53INP1 mRNA expression was also induced in our VIP and RA cotreatments but with a maximal increase by only 2 -fold, which is clearly less than the 7-fold observed after MYCN knockdown (26). This could be due to the fact that VIP and RA could have effects on multiple pathways and transcription factors regulating TP53INP1 expression. We also tested MDM2 expression, previously shown as another MYCN target gene, whose product prevents p53-mediated apoptosis (34). MDM2 mRNA expression was unchanged in our experiments (data not shown). This is in agreement with recent studies in which no variation of MDM2 expression was found after MYCN knockdown $(26,35)$.

Some studies have shown that the differentiating effect of RA can be enhanced when RA is used in combination with molecules such as histone deacetylase inhibitors or interferon- $\gamma$ $(36,37)$. A potentiating effect of RA and VIP has already been shown on tissue transglutaminase activity which is associated with apoptosis in neuroblastoma cells (38). Here, we report that this synergy between RA and VIP could also affect the expression of MYCN proteins and of SKP2 and TP53INP1 mRNA. Such synergistic effects could be related to studies demonstrating that VIP and RA systems are linked. Indeed, in the neuroblastoma cell lines, RA treatment stimulates VIP expression and increases the number of functional VIP binding sites (12,39). Furthermore, in the neuroblastoma SK-N-SH cell line, overexpression of the VIP receptor type VPAC1 results in an increased RA receptor expression (40).

The VIP-receptor system is involved in control of either proliferation or differentiation, depending on the type of cancer cells considered (8). Numerous studies allow the proposal that VIP or antagonists of this peptide could exhibit a therapeutic interest in various cancers. For example, VIP decreases growth of human small cell lung cancer cells in vitro and in vivo in mice (41), whereas VIP-antagonists lower growth of human ovarian or prostatic cancer cells in vivo in mice $(42,43)$. Moreover, other VIP-antagonists reduce or delay development of colon or mammary cancers in animal models of carcinogenesis induced chemically or genetically $(44,45)$. It 
was also observed that a VIP-antagonist enhances the effects of chemotherapeutic drugs in vitro and in vivo $(46,47)$. The present in vitro study, which demonstrated that VIP in association with RA decreased expression of MYCN proteins in a synergistic manner, extends the potential therapeutic interest of this neuropeptide to neuroblastoma cells with $M Y C N$ amplification, a genetic abnormality associated with poor prognosis in this type of cancer.

\section{Acknowledgements}

We thank D. Guyonnet for his excellent technical assistance with DNA sequencing and J. Habrioux for his helpful advice in the preparation of figures. This study was supported by grants from the Ligue Contre le Cancer (Comité Régional Poitou-Charentes) and from the 'Lions Club de Melle, France'. Lucie Chevrier and Stéphanie Cochaud were recipients of $\mathrm{PhD}$ fellowships from the Ligue Contre le Cancer (Comité Régional Poitou-Charentes) and from the French 'Ministère de l'Enseignement et de la Recherche', respectively.

\section{References}

1. Brodeur GM: Neuroblastoma: biological insights into a clinical enigma. Nat Rev Cancer 3: 203-216, 2003.

2. Plantaz D: Neuroblastoma a century after Pepper: which are the genes? Arch Pediatr 8: 917-921, 2001.

3. Weiss WA, Aldape K, Mohapatra G, Feuerstein BG and Bishop JM: Targeted expression of MYCN causes neuroblastoma in transgenic mice. EMBO J 16: 2985-2995, 1997.

4. Burkhart CA, Cheng AJ, Madafiglio J, et al: Effects of MYCN antisense oligonucleotide administration on tumorigenesis in a murine model of neuroblastoma. J Natl Cancer Inst 95: 1394-1403, 2003.

5. Ramsay G, Stanton L, Schwab M and Bishop JM: Human proto-oncogene $\mathrm{N}$-myc encodes nuclear proteins that bind DNA. Mol Cell Biol 6: 4450-4457, 1986.

6. Wenzel A, Cziepluch C, Hamann U, Schürmann J and Schwab M: The N-Myc oncoprotein is associated in vivo with the phosphoprotein $\operatorname{Max}(\mathrm{p} 20 / 22)$ in human neuroblastoma cells EMBO J 10: 3703-3712, 1991.

7. Strieder V and Lutz W: Regulation of N-myc expression in development and disease. Cancer Lett 180: 107-119, 2002.

8. Muller JM, Lelievre V, Becq-Giraudon L and Meunier AC: VIP as cell growth and differentiation neuromodulator role in neurodevelopment. Mol Neurobiol 10: 115-134, 1995.

9. Grosfeld JL: Risk-based management of solid tumors in children. Am J Surg 180: 322-327, 2000.

10. Muller JM, Philippe M, Chevrier L, Héraud C, Alleaume C and Chadéneau C: The VIP-receptor system in neuroblastoma cells. Regul Pept 137: 34-41, 2006.

11. Agoston DV, Colburn S, Krajniak KG and Waschek JA: Distinct regulation of vasoactive intestinal peptide (VIP) expression at mRNA and peptide levels in human neuroblastoma cells. Neurosci Lett 139: 213-216, 1992

12. Waschek JA, Muller JM, Duan DS and Sadée W: Retinoic acid enhances VIP receptor expression and responsiveness in human neuroblastoma cell, SH-SY5Y. FEBS Lett 250: 611-614, 1989.

13. Pence JC and Shorter NA: In vitro differentiation of human neuroblastoma cells caused by vasoactive intestinal peptide. Cancer Res 50: 5177-5183, 1990.

14. Hoshino M, Li M, Zheng LQ, Suzuki M, Mochizuki T and Yanaihara N: Pituitary adenylate cyclase activating peptide and vasoactive intestinal polypeptide: differentiation effects on human neuroblastoma NB-OK-1 cells. Neurosci Lett 159: 35-38, 1993.

15. Héraud C, Hilairet S, Muller JM, Leterrier JF and Chadéneau C: Neuritogenesis induced by vasoactive intestinal peptide, pituitary adenylate cyclase-activating polypeptide, and peptide histidine methionine in SH-SY5Y cells is associated with regulated expression of cytoskeleton mRNAs and proteins. J Neurosci Res 75: 320-329, 2004.
16. Hammerling U, Bjelfman C and Påhlman S: Different regulation of $\mathrm{N}$ - and $\mathrm{c}$ - myc expression during phorbol ester-induced maturation of human SH-SY5Y neuroblastoma cells. Oncogene 2: 73-77, 1987.

17. Kim SN, Kim SG, Park SD, Cho-Chung YS and Hong SH: Participation of type II protein kinase A in the retinoic acidinduced growth inhibition of SH-SY5Y human neuroblastoma cells. J Cell Physiol 182: 421-428, 2000.

18. O'Dorisio MS, Fleshman DJ, Qualman SJ and O'Dorisio TM: Vasoactive intestinal peptide: autocrine growth factor in neuroblastoma. Regul Pept 37: 213-226, 1992.

19. Grandinetti KB, Spengler BA, Biedler JL and Ross RA: Loss of one HuD allele on chromosome \#1p selects for amplification of the $\mathrm{N}$-myc proto-oncogene in human neuroblastoma cells. Oncogene 25: 706-712, 2006.

20. Mayer C, Popanda O, Zelezny O, von Brevern MC, Bach A, Bartsch $\mathrm{H}$ and Schmezer P: DNA repair capacity after gammairradiation and expression profiles of DNA repair genes in resting and proliferating human peripheral blood lymphocytes. DNA Repair (Amst) 1: 237-250, 2002.

21. Harris RG, White E, Phillips ES and Lillycrop KA: The expression of the developmentally regulated proto-oncogene Pax-3 is modulated by N-Myc. J Biol Chem 277: 34815-34825, 2002.

22. Tonelli R, Purgato S, Camerin C, et al: Anti-gene peptide nucleic acid specifically inhibits MYCN expression in human neuroblastoma cells leading to cell growth inhibition and apoptosis. Mol Cancer Ther 4: 779-786, 2005.

23. Manabe A, Iguchi-Ariga SM, Iizuka H and Ariga H: Transcriptional repression activity of N-MYC protein requires phosphorylation by MAP Kinase. Biochem Biophys Res Commun 219: 813-823, 1996.

24. Zaizen Y, Taniguchi S and Suita S: The role of cellular motility in the invasion of human neuroblastoma cells with or without N-myc amplification and expression. J Pediatr Surg 33: 1765-1770, 1998.

25. Chu PW, Cheung WM and Kwong YL: Differential effects of 9-cis, 13-cis and all-trans retinoic acids on the neuronal differentiation of human neuroblastoma cells. Neuroreport 14: 1935-1939, 2003.

26. Bell E, Lunec J and Tweddle DA: Cell cycle regulation targets of MYCN identified by gene expression microarrays. Cell Cycle 6: 1249-1256, 2007

27. Thiele CJ, Reynolds CP and Israel MA: Decreased expression of N-myc precedes retinoic acid-induced morphological differentiation of human neuroblastoma. Nature 313: 404-406, 1985.

28. Han G, Chang B, Connor MJ and Sidell N: Enhanced potency of 9-cis versus all-trans-retinoic acid to induce differentiation of human neuroblastoma cells. Differentiation 59: 61-69, 1995.

29. Lovat PE, Lowis SP, Pearson AD, Malcolm AJ and Redfern CP: Concentration-dependent effects of 9-cis retinoic acid on neuroblastoma differentiation and proliferation in vitro. Neurosci Lett 182: 29-32, 1994.

30. Lovat PE, Irving H, Annicchiarico-Petruzzelli M, et al: Retinoids in neuroblastoma therapy: distinct biological properties of 9-cis- and all-trans-retinoic acid. Eur J Cancer 33: 2075-2080, 1997.

31. Joshi S, Guleria R, Pan J, DiPette D and Singh US: Retinoic acid receptors and tissue-transglutaminase mediate short-term effect of retinoic acid on migration and invasion of neuroblastoma SH-SY5Y cells. Oncogene 25: 240-247, 2006.

32. Wang W, Nacusi L, Sheaff RJ and Liu X: Ubiquitination of p21Cip1/WAF1 by SCFSkp2: substrate requirement and ubiquitination site selection. Biochemistry 44: 14553-14564, 2005.

33. Tomasini R, Seux M, Nowak J, et al: TP53INP1 is a novel p73 target gene that induces cell cycle arrest and cell death by modulating p73 transcriptional activity. Oncogene 24: 8093-8104, 2005.

34. Slack A, Chen Z, Tonelli R, Pule M, Hunt L, Pession A and Shohet JM: The p53 regulatory gene MDM2 is a direct transcriptional target of MYCN in neuroblastoma. Proc Natl Acad Sci USA 102: 731-736, 2005.

35. Bell E, Premkumar R, Carr J, et al: The role of MYCN in the failure of MYCN amplified neuroblastoma cell lines to G1 arrest after DNA damage. Cell Cycle 5: 2639-2647, 2006.

36. Coffey DC, Kutko MC, Glick RD, et al: Histone deacetylase inhibitors and retinoic acids inhibit growth of human neuroblastoma in vitro. Med Pediatr Oncol 35: 577-581, 2000. 
37. Cetinkaya C, Hultquist A, Su Y, et al: Combined IFN-gamma and retinoic acid treatment targets the N-Myc/Max/Mad1 network resulting in repression of N-Myc target genes in MYCN-amplified neuroblastoma cells. Mol Cancer Ther 6: 2634-2641, 2007.

38. Goossens JF, Manechez D, Pommery N, Formstecher P and Hénichart JP: VIP potentiates retinoic-acid effect on tissue transglutaminase activity in human neuroblastoma, the SK-N-SH cells. Neuropeptides 24: 99-103, 1993.

39. Waschek JA, Lelievre V, Bravo DT, Nguyen T and Muller JM: Retinoic acid regulation of the VIP and PACAP autocrine ligand and receptor system in human neuroblastoma cell lines. Peptides 18: 835-841, 1997.

40. Balster DA, O'Dorisio MS, Albers AR, Park SK and Qualman SJ: Suppression of tumorigenicity in neuroblastoma cells by upregulation of human vasoactive intestinal peptide receptor type 1. Regul Pept 109: 155-165, 2002.

41. Maruno K, Absood A and Said SI: Vasoactive intestinal peptide inhibits human small-cell lung cancer proliferation in vitro and in vivo. Proc Natl Acad Sci USA 24: 1473-1478, 1998.

42. Chatzistamou I, Schally AV, Varga JL, Groot K, Armatis P and Bajo AM: Inhibition of growth and reduction in tumorigenicity of UCI-107 ovarian cancer by antagonists of growth hormonereleasing hormone and vasoactive intestinal peptide. J Cancer Res Clin Oncol 27: 645-652, 2001.
43. Plonowski A, Varga JL, Schally AV, Krupa M, Groot K and Halmos G: Inhibition of PC-3 human prostate cancers by analogs of growth hormone-releasing hormone $(\mathrm{GH}-\mathrm{RH})$ endowed with vasoactive intestinal peptide (VIP) antagonistic activity. Int J Cancer 98: 624-629, 2002.

44. Levy A, Gal R, Granoth R, Dreznik Z, Fridkin M and Gozes I: In vitro and in vivo treatment of colon cancer by VIP antagonists. Regul Pept 109: 127-133, 2002.

45. Moody TW, Dudek J, Zakowicz H, et al: VIP receptor antagonists inhibit mammary carcinogenesis in C3(1)SV40T antigen mice. Life Sci 74: 1345-1357, 2004.

46. Moody TW, Leyton J, Chan D, et al: VIP receptor antagonists and chemotherapeutic drugs inhibit the growth of breast cancer cells. Breast Cancer Res Treat 68: 55-64, 2001.

47. Gelber E, Granoth R, Fridkin M, Dreznik Z, Brenneman DE, Moody TW and Gozes I: A lipophilic vasoactive intestinal peptide analog enhances the antiproliferative effect of chemotherapeutic agents on cancer cell lines. Cancer 92: 2172-2180, 2001 